\title{
An Experimental System for Robotic Heart Surgery
}

\author{
H. Mayer ${ }^{1}$, I. Nágy ${ }^{1}$, A. Knoll ${ }^{1}$, E.U. Schirmbeck ${ }^{2}$ and R. Bauernschmitt ${ }^{2}$ \\ ${ }^{1}$ Chair of Robotics and Embedded Systems ${ }^{2}$ German Heart Center Munich \\ Technical University of Munich \\ $\{$ mayerh|nagy|knoll\}@in.tum.de, $\{$ schirmbeck|bauernschmitt\}@dhm.mhn.de
}

\begin{abstract}
In this paper we present an application of robotic systems in minimally invasive surgery. We have assembled a bi-manual telemanipulator capable of delicate operations. Each robotic arm has eight degrees of freedom which enables free manipulation via trocar kinematics. In addition minimally invasive instruments were equipped with strain gauge force sensors that can measure forces along three translational directions of the instrument's shaft. Forces are displayed to the user by means of two haptic devices which are also used for input. An endoscopic stereo-camera provides pictures from the region of operation to the user. In order to emulate a sterescopic impression, pictures can be displayed by means of either a head mounted display, a CRT-screen equipped with shutter technology or a $3 D$ video projection.

With this system we have assessed the quality of force feedback and we have performed typical operations known from cardiac surgery. We also have shown that it is possible to perform certain recurrent tasks (like knot tying) automatically. Within this context we have implemented two application examples. In the first example a surgical knot is recorded and afterwards replayed with increased speed at the same position. The second example is the instantiation of a predefined knot at an arbitrary position.
\end{abstract}

\section{Introduction}

In recent years, minimally invasive surgery (MIS) has become a promising option for a great number of medical interventions (like coronary heart surgery). However, apart from obvious advantages to the patient (including reduced tissue trauma and shorter recovery times), using this technique entails additional difficulties. The basic limitations are reduced sight and manipulability. This gap is closed by application of robotic systems. Available systems like the daVinci workstation (cf. [G. Guthart and J. Salisbury, 2000]) or the ZEUS system provide the surgeon with stereo vision of the operating environment and restore full control of the instruments. While having pioneered the field of endoscopic surgery, these systems also suffer from some deficiencies: they are telemanipulators with no direct position control (the control loop is implicitly closed by visual servoing of the surgeon) and they provide no possibility for force feedback. Both features are important in order to move the surgeon up in the control hierarchy, i.e. to implement "partial autonomy".

In order to overcome these hitches, two crucial issues have to be solved. One is inclusion of force sensory and feedback, the other is implementation of full Cartesian control of the end ef- 


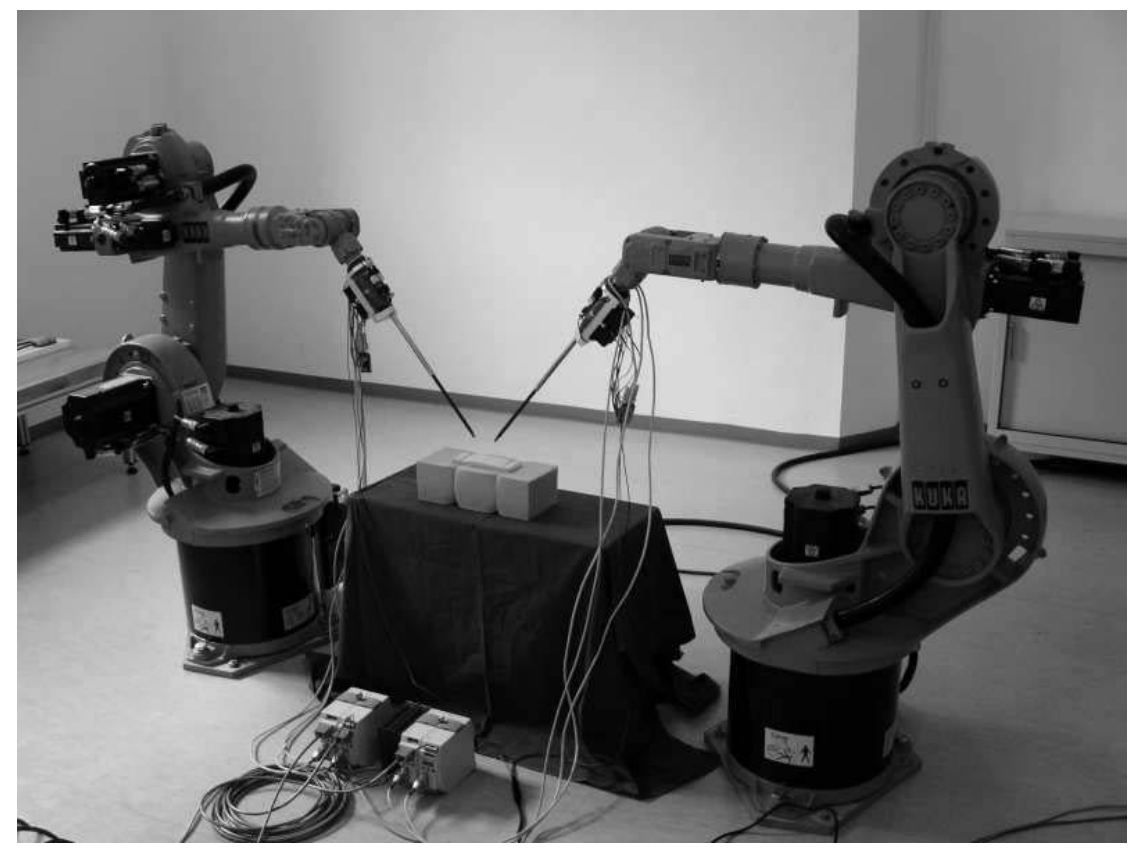

Figure 1. Setup of the Robotic Telemanipulator

fector. The latter is indispensable for calculating exact directions of forces in a known coordinate frame. Therefore one of our main research interests is the prototypical construction and evaluation of force sensory/feedback in realistic scenarios of robotic surgery. In particular we focus on instrumental suturing and knot-tying tasks, which are easy to apply when manually executed, but need a lot of experience to be performed via telemanipulation. These tasks, accomplished by human operators, were recorded, and after some processing steps they were autonomously executed.

A key role in this research project is taken by the adjustment of standard minimally invasive instruments (we took the one deployed with the $d a$ Vinci surgical system) for these challenges. Particular advantages of this setup with multi-purpose robots are high precision and stiffness, moderate costs and an advanced dynamic behavior. The latter could be exploited to perform sophisticated tasks in motion compensation (e.g. support for beating heart surgery as it was proposed in [T. Ortmaier et al. 2002], or compensation for respiratory motion of the ribs). The modular character of this setup simplifies the adaptation of the system to technical improvements (e.g. new surgical instruments). Another advantage is the fact that our manipulator is a robot under Cartesian control whose position can be adjusted precisely. Finally, the most important feature is the possibility for evaluation of force feedback in combination with endoscopic vision in robotic surgery. In order to make navigation easier, we additionally equipped the system with an endoscopic stereo camera system to observe the operation environment.

\section{Related Work}

Beside the already mentioned commercially telemanipulators daVinci and ZEUS, there exists a variety of systems for robotic surgery, implemented by other groups. At the University of California, Berkeley, a robotic system was developed, which has already been used to perform certain surgical tasks like suturing and knot-tying [M. Cavusoglu et al., 2003]. The Korean Advanced Institute of Science and Technology has developed a micro-telerobot system that also provides 
force feedback [D. Kwon et al., 1998]. In Germany two systems for robotic surgery were built at the Research Facility in Karlsruhe [U. Voges et al. 1997] and at the DLR in Oberpfaffenhofen [R. Konietschke et al., 2003]. While the first system provides no force feedback, the latter system is equipped with PHANToM devices for haptic display. There is also some work available dealing with analysis of knot-tying. At Johns Hopkins University, Kitagawa et al. [M. Kitagawa et al. 2002] have evaluated occurring forces during knot-tying. They did not measure forces directly at the instruments and during realistic operations, but with a specially designed measurement contrivance. Cao et al. [C. Cao et al. 1996] have analyzed a variety of surgical tasks (among other things knot tying) and decomposed them into subtasks. They did not include force measurement.

\section{Materials and Methods}

Like typical systems for robotic surgery, our setup comprises an operator-side master console for in-output and a patient-side robotic manipulator that directly interacts with the operating environment.

\subsection{Robotic Telemanipulator}

As one can see in figure 2, our system consists of two manipulators, which are controlled by two input devices. Each manipulator is composed of a Kuka KR 6/2 robot that bears a surgical instrument from Intuitive Surgical Inc. (deployed as part of the daVinci ${ }^{T M}$ surgical workstation). We have developed an adapter to link the robotic arm with the instrument. For security reasons we have equipped all flange adapters with magnetic security couplings. Those will be disengaged when forces are excerted which exceed a certain level and might cause harm on the instruments. The surgical instruments have three degrees of freedom. A micro-gripper at the distal end of the shaft can be rotated and adaptation of pitch and yaw angles is possible. Since the yaw angle of each of the two fingers of the gripper can be controlled separately, it is therefore possible to open and close the gripper. All movable parts of the gripper are driven by steel wires. Their motion is controlled by four driving wheels at the proximal end of the instrument, one four each degree of freedom (two for yaw of the fingers). In order to control the instrument, we have flanged servos to each driving wheel by means of an Oldham coupling. This guarantees instrument movement free of jerk. The servo controllers are connected via serial lines to a multiport card.

The Kuka robot has six degrees of freedom. Since the rotation of the robot's flange and the rotation of the instrument share one axis, our system finally has eight degrees of freedom. This redundancy renders the end effector possible to reach every position and orientation within the working space under restriction of so-called trocar kinematics.

Position and orientation of the manipulators are controlled by two PHANToM ${ }^{T M}$ devices from Sensable Inc. (see figure 2). This device is available in different versions with different capabilities. We have chosen the version PHANToM ${ }^{T M}$ Premium 1.5. It has a working space of approx. $20 \times$ $25 \times 40 \mathrm{~cm}$, which provides enough space to perform surgical procedures. The user controls a stylus pen that is equipped with a switch that can be used to open and close the micro-grippers.

\subsection{Optical System}

To enable proper telemanipulation it is indispensable to have a 3D-display providing a distinct vision of the region of interest. In order to allow for such a feature we equipped an additional robot 


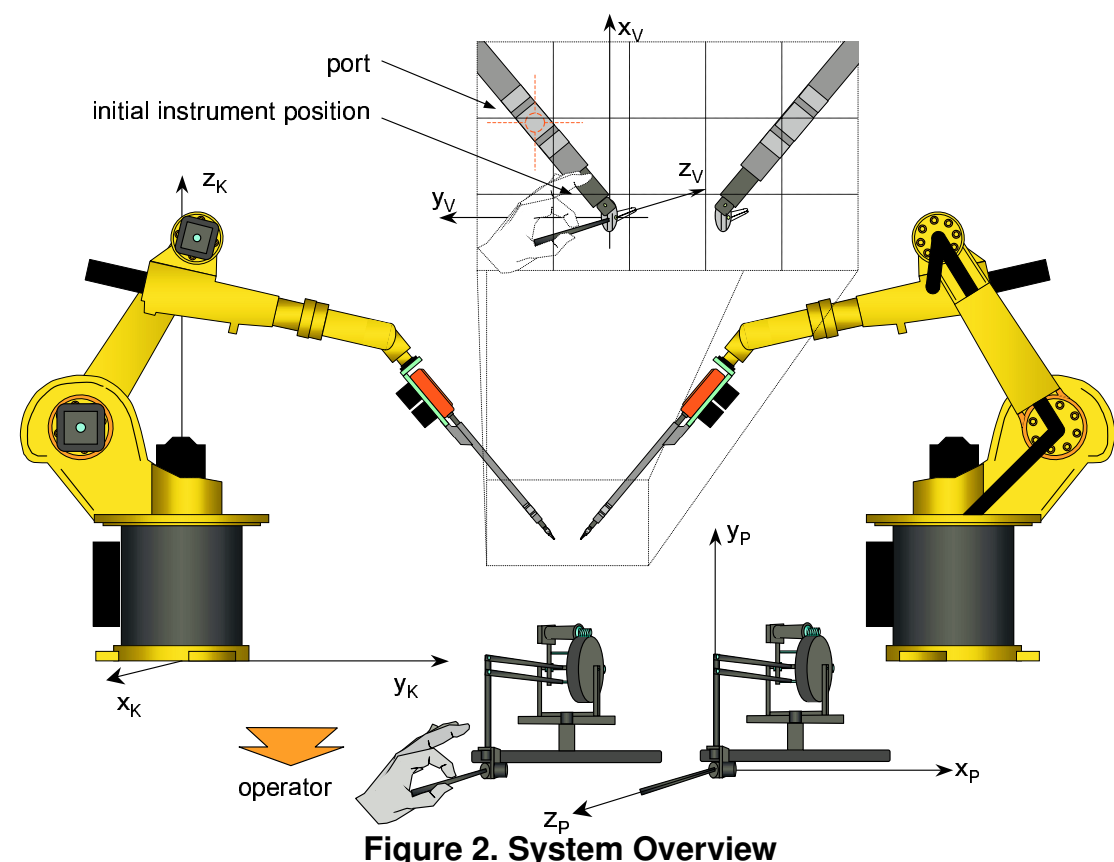

with a 3D endoscopic camera. Like the instruments, this camera can also be moved by means of trocar kinematics and can either be actively controlled by the operator or automatically tracked by the system.

Images taken from the stereo camera system can be displayed via three options. One is a head mounted display (HMD) that is part of our input console. Another possibility is to alternately display left and right images on a CRT-screen. In this case the operator has to wear shutter glasses, which are triggered by the output on the screen. A third option is the projection of the acquired pictures on a silver screen with two video projectors. The projectors have to be equipped with polarizing filters which are orthogonally arranged. Observers have to were glasses with an appropriate polarization for the corresponding eye. Tests have shown, that it is most convenient to work with the shutter system, because the operator is rendered able to see the hands and therefore gets a better hand-eye coordination. That is not possible with the HMD system. Unfortunately picture quality is not too good for none of the options mentioned above. Therefore we are currently working on a new modality based on optical systems for three dimensional interpretation of air photographs

\section{Results}

With the help of the described setup we have performed different tasks known from surgical practice and evaluated the impact of force measurement. Our hope is, that haptic feedback contributes to a better performance of systems for robotic surgery by preventing force-induced damages. Examples for such harms are breaking of thread material, ripping tissue and strangulate sutures.

\subsection{Preventing Suture Material Damage}

The tensile strength of absorbable and non-absorbable sutures is critical, both during and after surgical procedures. Given the breaking strengths of all used materials, we are able to prevent 
suture material damage by limiting the applicable forces to adequate maximum values. Figure 3 (left) shows the progression of forces while trying to break original surgical suture material, in this case Ethicon PROLENE (7/0, Polypropylene, not absorbable).

\subsection{Collision Detection}

Avoiding the collision of the instruments in robot assisted minimally invasive surgery is not an easy task. Therefore a symbolic representation of the whole robotic system, including both the instruments and the arms, would be necessary. Furthermore exact position control and a collision detection software subsystem are indispensable. Most setups however do not provide the above mentioned infrastructure. A human operator will easily avoid instrument collisions, but in an autonomous mode other solutions are necessary. A force controlled setup will not prevent collisions, but an early detection can avoid damages of the instruments. The right side of figure 3 shows the forces recorded during an instrument collision. The instrument velocities were within ranges typical to this scenario. We observe, that the highest peak ( $Y$-force component of the left instrument) arises within approximately $35 \mathrm{~ms}$. With a robot arm interpolation of $12 \mathrm{~ms}$ there are nearly 3 interpolation periods to react when such a situation occurs, providing a satisfactory collision interception.
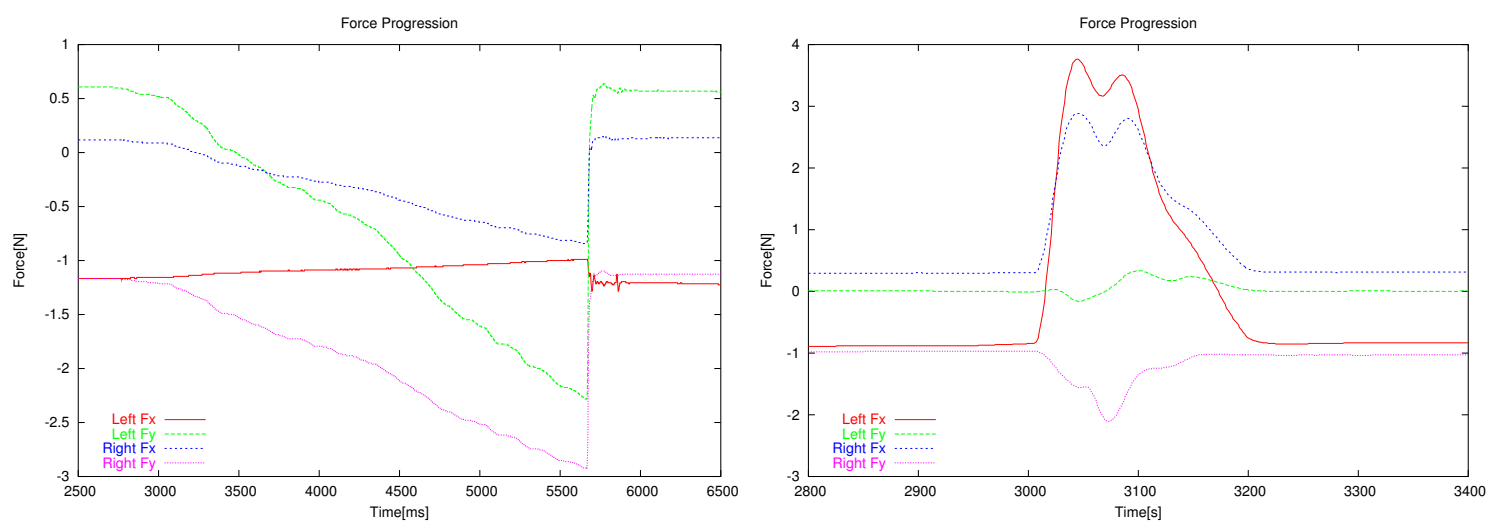

Figure 3. Breaking Ethicon $7 / 0$ (left) and Instrument Collision (right)

\subsection{Automated Knot Tying}

With the help of the setup described above, we have performed several knot-tying tasks and recorded both, force progression and the corresponding trajectories (described by position and orientation of the instruments). Due to inevitable physiological tremor of the human operator, the acquired trajectories exhibit some noise. Therefore two-stage preprocessing was applied to the raw data. The first stage comprises sliding window averaging, the second stage approximates the smoothed data with natural cubic splines.

Our first experiment was replay of an original sample with no smoothing and approximation applied. Since our system features a high repeat accuracy, this procedure was performed very reliable. The only prerequisite is positioning the needle at a known place. Since we leave the needle placement to the surgeon and we know the geometry of our system, we can always exactly locate the corresponding position. Due to exact kinematics, execution of up to double speed has raised no difficulties. As our objective is not restricted to acceleration, we also want to generate optimized trajectories with respect to smoothness and path planning. Therefore we have applied spline approximation to the raw data. This results in a symbolic representation of the trajectory in the form of a 
parametric space-curve. Before applying the generated curve to the real system, collision avoidance has to be guaranteed, since overmodified paths can contingently result in instrument collision.

We have used this spline approximated trajectory to perform a second experiment. The trajectory is not replayed exactly at the coordinates where it was recorded, but at an arbitrary position. This position is indicated by the operator with the tip of the instrument. To execute a knot at an arbitrary position, the user has to grasp the needle after piercing it through the point where a suture has to be put. Now the system knows where to start knot-tying and a rigid transformation of the previously recorded and smoothed trajectory can be calculated. After transforming it, the knot-tying trajectory can be applied at the corresponding position. Currently transformations are restricted to translations, but we are planning to integrate rotations and scaling in the near future.

\section{Conclusion}

We have presented a novel approach of a robotic system for minimally invasive surgery. The main purpose of the system is evaluation of force feedback. We found out that performance of certain surgical tasks like knot tying will profit from this feature. Forces are measured at the surgical instruments and fed back into the surgeon's hands using multi-dimensional haptic styluses. For future evaluation we are planning long-term tests to find out if force feedback can prevent surgeon's fatigue. The current arrangement of input devices, however, is not very comfortable. Therefore we are planning to test different rearrangements of this setup and to develop an own input instrument to replace the stylus pen.

In addition we have implemented full cartesian control of the end effectors. This feature was exploited for two realistic application examples. Knot-tying tasks were recorded and replayed with higher speed and at arbitrary positions.

Integration of force feedback and stereo vision, as it is offered by the system, will improve accuracy and reduce the time needed for operations. In addition it will help to avoid collateral tissue trauma and lead to a reduction of stress of the surgeon. This could result in a wider acceptance of robotic surgery by both, patients and surgeons. The system's software interfaces and mechanical set-up descriptions are freely available to enable other research groups to participate in the development.

\section{References}

[G. Guthart and J. Salisbury, 2000] G.S. Guthart and J.K. Salisbury (2000), The Intuitive ${ }^{T M}$ Telesurgery System: Overview and Application, IEEE International Conference on Robotics and Automation, San Francisco CA, USA.

[T. Ortmaier et al. 2002] T. Ortmaier, M. Groeger and G. Hirzinger (2002), Robust Motion Estimation in Robotic Surgery on the Beating Heart, Computer Assisted Radiology and Surgery (CARS), Paris, France

[M. Cavusoglu et al., 2003] Cavasoglu M. et al. (2003), Robotics for Telesurgery: Second Generation Berkeley/UCSF Laparoscopic Telesurgical Workstation and Looking towards the Future Applications, Industrial Robot, Special Issues on Medical Robotics, Vol. 30, no. 1

[D. Kwon et al., 1998] D. Kwon et al. (1998), Microsurgical Telerobot System, IEEE/RSJ International Conference on Intelligent Robots and Systems, pp. 945-950

[R. Konietschke et al., 2003] R. Konietschke et al. (2003), Optimal Design of a Medical Robot for Minimally Invasive Surgery, 2. Jahrestagung der Deutschen Gesellschaft fuer Computer- und Roboterassistierte Chirurgie (CURAC), Nuernberg, Germany

[U. Voges et al. 1997] U. Voges et al. (1997), Evaluation of ARTEMIS: the Advanced Robotics and Telemanipulator System for Minimally Invasive Surgery, Proceedings IARP 2nd Workshop on Medical Robotics, pp. 137-148

[M. Kitagawa et al. 2002] M. Kitagawa, A. M. Okamura et al. (2002), Analysis of Suture Manipulation Forces for Teleoperation with Force Feedback, Technical Report, Johns Hopkins University, Baltimore MD, USA

[C. Cao et al. 1996] C. Cao, C. MacKenzie and S. Payandeh (1996), Task and motion analyses in endoscopic surgery, Proceedings ASME Dynamic Systems and Control Division, pp. 583-590, Atlanta, USA 